\title{
Interpersonal Dependency Inventory: Its Construct Validity and Prediction of Dysphoric Mood and Life Functioning via Negative Life Events
}

\author{
Masayo $\mathrm{Uji}^{{ }^{*}}$, Yukihiro Takagishi ${ }^{2}$, Keiichiro Adachi ${ }^{3}$, Toshinori Kitamura ${ }^{4}$ \\ ${ }^{1}$ Department of Bioethics, Kumamoto University Graduate School of Life Sciences, Kumamoto, Japan \\ ${ }^{2}$ Department of Human Psychology, Kansai University of International Studies, Miki, Japan \\ ${ }^{3}$ Department of Psychology, Kobe Shoin Women's University, Kobe, Japan \\ ${ }^{4}$ Kitamura Mental Health Institute Tokyo, Akasaka, Japan \\ Email: "ujimasayo@hotmail.co.jp
}

Received October $5^{\text {th }}, 2013$; revised November $2^{\text {nd }}, 2013$; accepted November $28^{\text {th }}, 2013$

Copyright (C) 2013 Masayo Uji et al. This is an open access article distributed under the Creative Commons Attribution License, which permits unrestricted use, distribution, and reproduction in any medium, provided the original work is properly cited. In accordance of the Creative Commons Attribution License all Copyrights (C) 2013 are reserved for SCIRP and the owner of the intellectual property Masayo Uji et al. All Copyright (C 2013 are guarded by law and by SCIRP as a guardian.

Previous Dependency Inventory (JIDI) as well demonstrates how the interpersonal attitudes assessed by the JIDI generate interpersonal negative life events (NLEs) and therefore results in dysphoric moods and poor life functioning. The subjects of this study were 467 Japanese university students. The JIDI was administered at Time 1, and the Clinical Outcomes in Routine Evaluation-Outcome Measure (CORE-OM) was administered at Time 2 for the purpose of assessing dysphoric mood and life functioning. NLEs occurring between Times 1 and 2 were evaluated. The three-factor structure of the JIDI was confirmed by confirmatory factor analysis. The structural equation modeling demonstrated that of the three factors, only one, "emotional reliance", predisposed individuals to dysphoric mood and poor life functioning, both directly and indirectly, via interpersonal NLEs. Although the other two factors did not increase vulnerability to interpersonal NLEs or dysphoric mood, "lack of social self-confidence" worsened general functioning, and "assertion of autonomy" led to poor functioning in close relationships, both directly. Furthermore, "assertion of autonomy" decreased anxiety levels. As to gender differences, simultaneous analysis of multi-groups showed that female subjects were more likely to become anxious following interpersonal NLEs.

Keywords: Japanese Version of the Interpersonal Dependency Inventory; Factor Structure; Negative Life Events; Mental Health Problems

\section{Introduction}

The Interpersonal Dependency Inventory Japanese Short Form (JIDI) was developed by McDonald-Scott (1988) by modifying the original Interpersonal Dependency Inventory (IDI: Hirschfeld et al., 1977). Among the 48 items in the original version, those for which it was difficult to define corresponding Japanese expressions were removed from the JIDI, resulting in 23 JIDI items. The reliability and internal consistency of the JIDI were confirmed (McDonald-Scott, 1988). Despite the differences between the IDI and JIDI in terms of language and number of items, the two instruments are both able to assess the concepts described below.

Hirschfeld et al.'s (1977) concept of interpersonal dependency derives from the psychoanalytic theory of object relations, the social learning theory of dependency, and the ethological theory of attachment, resulting in the ability of the developed inventory (the IDI) to evaluate both expressed behaviors and intrapsychic elements such as thoughts, beliefs, and feelings. As with the original version, the JIDI consists of three factors, "Corresponding author. "emotional reliance", "lack of social self-confidence", and "assertion of autonomy".

According to Hirschfeld et al. (1977), the first factor, "emotional reliance", reflects the notions of attachment and dependency: "Attachment-related items express a wish for contact with and emotional support from specific other persons, as well as expressing a dread of loss of that person. Dependency-related items involve a general wish for approval and attention from others" (Hirschfeld et al., 1977). Examples of items in this category include "I need to have some person who puts me above all others", and "I have always had a terrible fear that I will lose the love and support of people I desperately need" (Hirschfeld et al., 1977).

The items that comprise the second factor, "lack of social self-confidence", express one's "wishes for help in decisionmaking, in social situations, and in taking initiative". McDonald-Scott (1988) further explains the two psychological processes behind these behaviors. The first is the fear of whether specific others will give their approval, and the other is difficulty with autonomous behaviors due to a lack of self-confi- 
dence in one's own judgment. Sample items are "When I have a decision to make, I always ask for advice", and "It is hard for me to ask someone for a favor" (Hirschfeld et al., 1977).

The items that constitute the third factor, "assertion of autonomy", "tend to deny either attachment or dependency". McDonald (1988) wrote that individuals who score highly on this factor have an intense need to rely on others; however, because of the fear of rejection, these emotional needs are either suppressed or denied. Examples of inventory items include, "When I am sick, I prefer that my friends leave me alone", and "I don't need anyone" (Hirschfeld et al., 1977). This category can be regarded as a defense against dependence shown by the significant correlation between "assertion of autonomy" and "interpersonal sensitivity" (Hirschfeld et al., 1977). Interpersonal sensitivity indicates fear of disapproval or rejection.

This study had three aims. The first was to confirm the hypothesized three-factor structure of the JIDI (Figure 1).

The second was to determine whether the three factors of the JIDI cause dysphoric mood and poor life functioning. With regard to the second aim, the relationship between these three factors and mental health indices has been demonstrated by calculating the correlations between them (Hirschfeld et al., 1977; McDonald-Scott, 1988). Previous researchers (Hirschfeld et al., 1977; McDonald-Scott, 1988) demonstrated almost the same results: the two factors "emotional reliance" and "lack of social self-confidence" were related to negative mental health indices such as depression and anxiety. This relation was not found for "assertion of autonomy".

In this study, we focused on the process whereby high "emotional reliance" or high "lack of social self-confidence" results in negative mental health outcomes. More specifically, we used structural equation modeling (SEM) to examine whether these processes are associated with negative life events (NLEs).

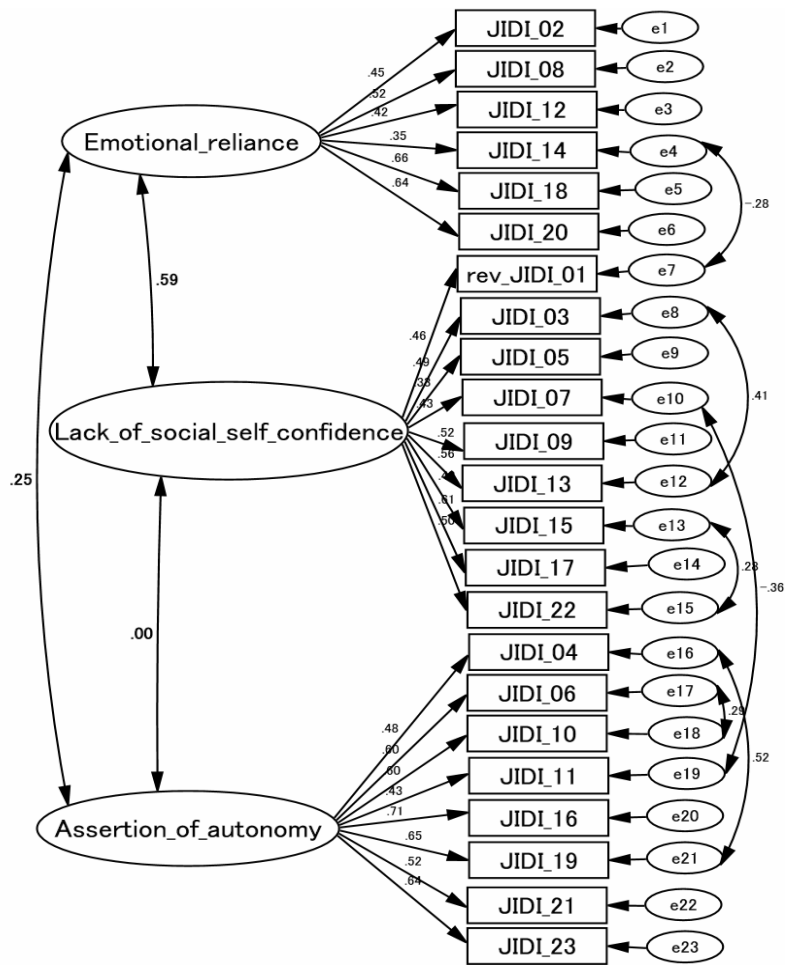

Figure 1.

The three-factor structure model of the JIDI.
Several studies have examined the tendency of specific personality types to generate NLEs (Bolger \& Schilling, 1991; Daley, Hammen, Davila, \& Burge, 1998; Magnus, Diener, Fujita, \& Pavot, 1993; McLennan \& Bates, 1993; Ormel \& Wohlfarth, 1991) as well as depression (Daley et al., 1997; Davila, Hammen, Paley, \& Daley, 1995; Hammen, 1991). Personality describes a wide range of mental activities, such as cognitions, emotions, and attitudes, and also influences behavioral patterns. Simons, Angell, Monroe, and Thase (1993) noted that cognitive factors influence not only the definition and severity evaluation of NLEs, but also the actual triggering of NLEs by way of an individual's behavior. Thus an individual's personality influences how the NLE is defined, how its intensity is evaluated, the nature of the emotional reaction it elicits, and what kinds of coping behaviors are chosen. To achieve the second aim of this study, we examined whether interpersonal dependency as a personality trait impacts one's tendency to generate interpersonal NLEs, and consequently, to result in negative mental health outcomes.

Concerning the pathways from each JIDI factor to mental health indices, we hypothesized that people with either high "emotional reliance" or "lack of social self-confidence" provoke interpersonal NLEs (Figure 2: W1 and W3) that eventually undermine mental health, for the following reasons. With regard to the first factor, excessive "emotional reliance" would result in the experiencing of intense needs and expectations that others cannot fulfill. This would increase the individual's levels of dependency, manipulation, and control of specific others. Feeling overwhelmed, the specific others may then keep their distance from him or her. The individual may experience feelings of rejection, resulting in an interpersonal NLE.

Concerning the second factor, if one is lacking in social self-confidence, they may repress their opinions in order to maintain the favor of others. As a result, they may not be respected, and may sometimes be disregarded, because they seem to be too submissive toward others. This in turn might lead to stress in interpersonal relationships, and possibly the development of resentment toward others.

In contrast to the two factors mentioned above, the third factor, "assertion of autonomy", would offer protection from interpersonal NLEs (Figure 2: W5) because it involves avoiding interaction with others. However, an alternative hypothesis is possible. Beck's (1983) concept of autonomy seems to be

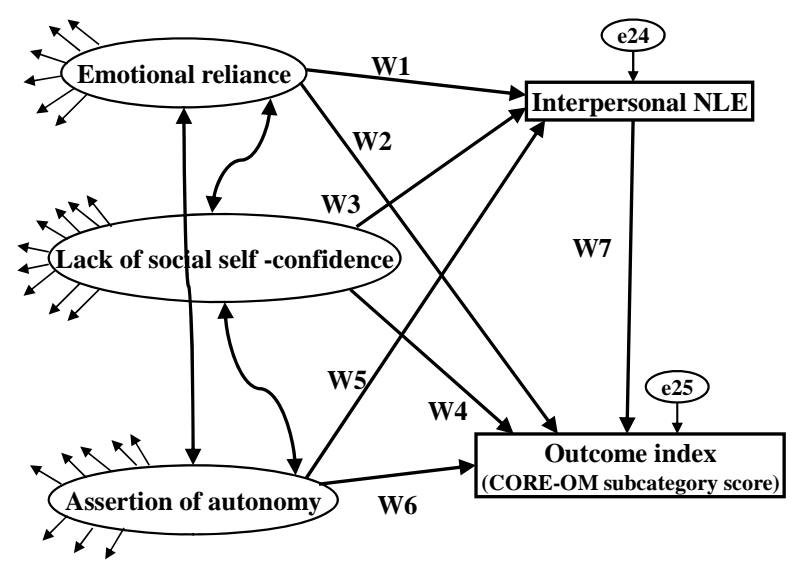

Figure 2.

The hypothesis pathway from each JIDI factor to negative mental health outcomes. 
closely related to the JIDI's "assertion of autonomy" in terms of an individual's attitudes. Beck (1983), however, does not specify whether or not he sees autonomy as a defense against dependency, and writes that although an individual in "the autonomous mode" has his or her "own set of internal standards, goals, criteria for achievement", as well as a "high level of selfconfidence and self-esteem", he or she is "less sensitive to others' needs and wishes" and tends to be "dogmatic" and "authoritarian." Some "assertion of autonomy" items in the JIDI, such as "I hate it when people offer me sympathy" or "I rely on myself", infer rejection or hatred for affectionate care rather than independence. Based on these characteristics, people who score highly on this factor might have difficulty recognizing their need for others, and therefore also lack empathy for others' feelings. These characteristics suggest that such individuals would encounter antipathy from others. Indeed, Daley et al. (1997) used Beck's Sociotropy-Autonomy Scale (SAS) to demonstrate that in contrast to an individual with sociotropy, one with high autonomy tends to cause interpersonal stress in others even after psychiatric status is taken into account. Therefore, we hypothesized that as with the first and second factors, the third factor, "assertion of autonomy", would cause interpersonal NLEs.

We also hypothesized that there are direct pathways from all three factors to negative mental health outcomes (Figure 2: W2, W4, W6). The third factor, "assertion of autonomy," could also cause dysphoric mood and poor life functioning, because individuals scoring highly on this factor are not able to seek help from others when they need it. This makes it difficult for them to accomplish their goals, leading to feelings of anxiety and depression as well as poor life functioning (Figure 2: W6). Indeed, there is also evidence of a close relationship between autonomy and undermined mental health. For instance, Bieling, Beck, and Brown (2000) write that the concept of autonomy consists of two factors: first, "sensitivity to others' control", and second, "independent goal attainment". The JIDI's "assertion of autonomy" seems to be closely related to the first factor, which was presented as having a positive correlation with psychopathology by Bieling et al. (2000). In this study, we not only examined correlations, but also aimed to verify the causal relationship between "assertion of autonomy" and impaired mental health (Figure 2: W6).

The third aim of this study was to assess the gender differences regarding the above pathways. Prior studies have shown that women are more likely to report interpersonal stresses than men (Mezulis, Funasaki, Charbonneau, \& Hyde, 2010; Rudolph \& Hammen, 2003; Kendler, Thornton, \& Prescott, 2001). From this, it can be assumed that either women are more sensitive in recognizing interpersonal conflicts, or they actually experience interpersonal NLEs more often. If the former is the case, the above hypothesized pathway from interpersonal dependency to negative mental health through interpersonal NLEs could be identified more clearly among women. On the other hand, men might be less likely to recognize interpersonal NLEs, and therefore among male subjects it might not be difficult to identify an indirect pathway from JIDI subscales to negative mental health indices by way of interpersonal NLEs. Rather, we might only find a direct pathway.

An additional hypothesis is that women are more likely to place an emphasis on interpersonal relationships, and socially, it is more acceptable for them to depend on others. Therefore, we theorized that they would score more highly than male sub- jects on assessments of "emotional reliance". Furthermore, negative mental health indices could be more strongly influenced by JIDI subscales as well as interpersonal NLE scores among women as compared to men.

To summarize, the purposes of this study were as follows:

1) to confirm the hypothesized three-factor structure of the JIDI using confirmatory factor analysis (CFA);

2) to examine whether the factors that comprise the JIDI caused dysphoric mood and poor life functioning directly or indirectly via interpersonal NLEs, using SEM; and

3 ) to determine whether the pathways varied with gender.

\section{Methods}

\section{Procedures}

This study consisted of self-reported questionnaires conducted on two occasions. The JIDI (evaluated on the first occasion), and Clinical Outcomes in Routine Evaluation-Outcome Measure (CORE-OM) and an assessment of interpersonal NLE (evaluated on the second occasion), were included in the questionnaires. In this study, Time 1 represents the first occasion and Time 2 represents the second occasion. The interval between the Time 1 and Time 2 was six weeks.

\section{Participants}

Four hundred sixty-nine students from two universities, two nursing schools, and one vocational school in Kumamoto attended both Times 1 and 2. Of these students, two declined to participate, resulting in a study population of 467 students. Questionnaires were distributed to the students during classes. The subjects' mean (SD) age was 19.3 (3.5), and the numbers of female and male respondents were 342 and 125, respectively. The research protocol was approved by the Ethical Committee of Kumamoto University (Institutional Review Board). Participation was voluntary, and subjects were assured of anonymity. For the purpose of anonymity, aliases were used.

\section{Measurements}

\section{JIDI (McDonald-Scott, 1988)}

As noted in the introduction, the JIDI was developed as a revision of the IDI (Hirschfeld et al., 1977). The JIDI consists of 23 items in total: six for "emotional reliance", nine for "lack of social self-confidence", and eight for "assertion of autonomy". Each item is rated from 1 (strongly disagree) to 5 (strongly agree). Therefore, the total score of the JIDI ranges from 23 to 115. Higher scores indicate greater identification with each of the three factors. McDonald-Scott (1988) attested to the JIDI's reliability and internal consistency.

\section{Clinical Outcomes in Routine Evaluation-Outcome Measure (CORE-OM: Evans et al., 2002)}

The CORE-OM was developed as a standardized brief outcome measure for use in both routine clinical practice and psychotherapy research (Evans et al., 2002). The reliability and validity of the Japanese version of the CORE-OM have been demonstrated (Uji, Sakamoto, Adachi, \& Kitamura, 2012). Of the 34 items, eight dysphoric mood items (four anxiety and four depression) and twelve life functioning items (four close functioning, four general functioning, and four social functioning) were chosen for this study. Each item is rated from 1 (strongly 
disagree) to 5 (strongly agree). Higher scores indicate more serious mental problems. Therefore, the total scores of each subcategory (anxiety, depression, close functioning, general functioning, and social functioning) range from 4 to 20 .

\section{Interpersonal NLEs}

The following ad hoc question was used to assess interpersonal NLEs: "Please evaluate the distressful experiences you had in the past two weeks in terms of relationships with the following people: 1) your friends, 2) your boyfriend/girlfriend, 3) your mother, 4) your father, 5) faculty members, and 6) your coworkers". Each interpersonal relationship was evaluated with a score ranging from 0 (no distressful experience) to 100 (extremely distressful experience). Therefore, the total score of the interpersonal NLE assessment ranges from 0 to 600 .

\section{Statistical Analysis}

To examine whether the JIDI fit the hypothesized three-factor model (Figure 1), we conducted a CFA. Furthermore, to assess whether our hypothesized pathways from each JIDI factor to negative mental health outcomes (Figure 2) fit the data, we conducted SEM. To examine the gender influence on the parameters in Figure 2, simultaneous analyses of multi-groups were conducted. A critical ratio with a magnitude of 1.96 or more indicates a significant difference $(p<0.05)$ in the parameters between the pair of subgroups.

SPSS version 21.0 and Amos version 21.0 were used for CFA and SEM. The fit of the hypothesized models to our data (Figures 1 and 2) was evaluated using the following indices: Goodness of Fit Index (GFI), Adjusted Goodness of Fit Index (AGFI), and Root Mean Square Error of Approximation (RMSEA) (Arbuckle \& Wothke, 1995).

\section{Results}

\section{CFA of the JIDI}

The model that presumed the covariance between "lack of social self-confidence" and "assertion of autonomy" to be zero demonstrated the best fit (GFI $=0.88$, AGFI $=0.85$, RMSEA $=$ 0.08 ). The covariance between "emotional reliance" and "lack of social self-confidence," as well as that between "emotional reliance" and "assertion of autonomy," were significant $(p<$ $0.01)$.

\section{The Influence of Gender on the JIDI Subscale Scores}

There was no gender influence identified on any JIDI subscale score (Table 1).

\section{Pearson's Correlations of Each JIDI Subscale with Mental Health Consequences as Well as with Interpersonal NLEs}

Regarding the correlations between each JIDI subscale and mental health consequences, almost all the CORE-OM subcategory scores were found to be significantly correlated with the JIDI subscales, the exception being non-significant correlations between "assertion of autonomy" and both anxiety and general functioning (Table 1).

Concerning the correlations between each JIDI subscale and interpersonal NLEs, only "assertion of autonomy" did not have a significant correlation with interpersonal NLEs (Table 1).

\section{The Pathways from JIDI Subscales to Negative Mental Health Outcomes}

The results of SEM are shown in Table 2. "Emotional reliance" was the only factor that generated interpersonal NLEs (Figure 2: W1, standardized causal coefficient (scc) was 0.34 $0.35(p<0.001))$, which in turn resulted in increased anxiety and depression levels and decreased close and social functioning (Figure 2: W7, sccs were $0.20(p<0.001), 0.18(p<0.001)$, $0.16(p<0.001)$, and $.22(p<0.001)$, respectively). There were also direct pathways from "emotional reliance" to these mental health outcome indices (Figure 2: W2) (scc was 0.43 ( $p<$ $0.001)$ for anxiety, $0.37(p<0.001)$ for depression, $0.23(p<$ $0.001)$ for close functioning, and $0.34(p<0.001)$ for social functioning). In contrast, "emotional reliance" had no impact on general functioning, either directly or indirectly. "Lack of social self-confidence" directly lowered general functioning (Figure 2: W4, scc was $0.41, p<0.001)$. In comparison, "assertion of autonomy" lowered close functioning (Figure 2: W6, scc was $0.18(p<0.001)$, whilst alleviating anxiety (Figure 2: W6, scc was $-0.15, p=0.006)$. The fitness of the hypothesized model to our data (Figure 2) for each mental health index was acceptable: GFI: 0.86 - 0.87, AGFI: 0.83 - 0.84, and RMSEA: 0.07). Simultaneous analyses of multi-groups were conducted to determine the gender influence on each parameter. The only difference between the genders was that the influence of interpersonal NLEs on anxiety was significantly higher among female respondents (critical ratio: 2.29).

Table 1.

Differences in mean scores (SD) between females and males and correlations between JIDI subscale scores and CORE-OM subscale scores.

\begin{tabular}{|c|c|c|c|c|}
\hline & & Emotional reliance & Lack of social self-confidence & Assertion of autonomy \\
\hline \multirow{2}{*}{ Mean scores (SD) } & male & $16.2(4.5)$ & $27.2(6.3)$ & $16.7(6.1)$ \\
\hline & female & $15.5(4.4)$ & $27.4(6.3)$ & $15.9(5.9)$ \\
\hline \multirow[t]{3}{*}{$\mathrm{p}$ value } & & 0.11 & 0.68 & 0.22 \\
\hline & anxiety & $0.37^{* *}$ & $0.23^{* *}$ & -0.04 \\
\hline & depression & $0.35^{* *}$ & $0.24^{* *}$ & $0.15^{* *}$ \\
\hline \multirow{3}{*}{$\begin{array}{l}\text { Correlations with the CORE-OM subcategory } \\
\text { scores }\end{array}$} & social functioning & $0.33^{* *}$ & $0.20^{* *}$ & $0.18^{* *}$ \\
\hline & general functioning & $0.20^{* *}$ & $0.33^{* *}$ & 0.03 \\
\hline & close functioning & $0.24^{* *}$ & $0.15^{* *}$ & $0.23^{* *}$ \\
\hline \multicolumn{2}{|c|}{ Correlation with Interpersonal NLEs } & $0.28^{* *}$ & $0.15^{* *}$ & 0.04 \\
\hline
\end{tabular}

Note: ${ }^{* *} p<0.01$. 
Table 2.

SEM results.

\begin{tabular}{ccccccccccccccc}
\hline $\begin{array}{c}\text { CORE-OM } \\
\text { subcategory }\end{array}$ & $\mathrm{N}$ & \multicolumn{1}{c}{ standardized causal coefficient (scc) in Figure 2} & & & $\begin{array}{c}\text { Absolute value of critical ratio }>1.96 \text { between male } \\
\text { and female groups in Figure } 2\end{array}$ \\
\hline & & W1 & W2 & W3 & W4 & W5 & W6 & W7 & GFI & AGFI & RMSEA & \\
Anxiety & 405 & $0.35^{* * *}$ & $0.43^{* * *}$ & 0 & 0 & 0 & $-0.15^{* *}$ & $0.20^{* * *}$ & 0.87 & 0.84 & 0.07 & W7: female (scc was 0.26$)>$ male (scc was 0.07$)$ \\
Depression & 402 & $0.35^{* * *}$ & $0.37^{* * *}$ & 0 & 0 & 0 & 0 & $0.18^{* * *}$ & 0.87 & 0.84 & 0.07 & none \\
Close functioning & 405 & $0.35^{* * *}$ & $0.23^{* * *}$ & 0 & 0 & 0 & $0.18^{* * *}$ & $0.16^{* * *}$ & 0.87 & 0.83 & 0.07 & none \\
General functioning & 406 & $0.35^{* * *}$ & 0 & 0 & $0.41^{* * *}$ & 0 & 0 & 0 & 0.86 & 0.83 & 0.07 & none \\
Social functioning & 405 & $0.34^{* * *}$ & $0.34^{* * *}$ & 0 & 0 & 0 & 0 & $0.22^{* * *}$ & .87 & .84 & 0.07 & none
\end{tabular}

Note: ${ }^{* *} p<0.01,{ }^{* * *} p<0.001$.

\section{Discussion}

\section{CFA of the JIDI}

The result of the CFA supported the hypothesized three-factor structure of the JIDI. Despite the fact that "assertion of autonomy" showed no significant correlation with "lack of social self-confidence", it was significantly correlated with "emotional reliance". Therefore, as McDonald-Scott (1988) noted, an intense need for others could underlie both "emotional reliance" and "assertion of autonomy". The difference is that an individual with high "emotional reliance" is able to recognize their needs, while an individual with high "assertion of autonomy" is apt to deny their needs because of fear of disapproval and rejection by significant others.

The other significant correlation, between "emotional reliance" and "lack of social self-confidence", can be interpreted as follows: people with high levels of "emotional reliance" always need others' approval and care, which means that they do not have sufficient "social self-confidence". If they have high "social self-confidence" and no need for others' approval, then they do not have to be so reliant on others.

\section{Lack of Significant Influences of Gender on the JIDI Subscales}

Contrary to our hypothesis, there were no gender differences in the evaluation of the JIDI subscales. In traditional Japanese society, men were required to be masculine and assertive, and women feminine and passive. However, in contemporary times, these socially required gender roles are less widely recognized, which has hypothetically led to the lack of differences between genders in JIDI subscale scores.

\section{The Direct and Indirect Influences of "Emotional Reliance” on Dysphoric Mood and Poor Life Functioning}

As predicted, "emotional reliance" triggered interpersonal NLEs, which in turn led to dysphoric mood and poor life functioning. The exception was the lack of impact of "emotional reliance" on general functioning. An individual with high "emotional reliance" seems to be deficient in the "capacity to be alone" (Winnicott, 1958), and seeks instant gratification through a dependence on others. These attitudes and behaviors may provoke interpersonal NLEs, and eventually result in dysphoric mood and poor life functioning. Therefore, the deteriorated mental health experienced by those with high "emotional reliance" can be partially due to these individuals' cognitive styles, attitudes, and behaviors. The direct pathways from "emotional reliance" to dysphoric mood and poor life functioning were also determined.

\section{Absence of Significant Influences of "Lack of Social Self-Confidence” on Generation of Interpersonal NLEs or Dysphoric Mood, and Its Negative Impact on “General Functioning Level”}

Despite the significant Pearson's correlations of "lack of social self-confidence" with anxiety, depression, social functioning, close functioning and interpersonal NLEs, the SEM results showed that "lack of social self-confidence" provoked none of these. The reason for this discrepancy may be that SEM takes into account all factor relationships, such as correlations within the three JIDI subscales. There would be an alternative reason why "lack of social self-confidence" did not influence interpersonal NLEs: an individual with severe "lack of social self-confidence" tends to be ego-centric, and thus does not have the mental capacity to recognize interpersonal conflicts.

Concerning life functioning, individuals demonstrating "lack of social self-confidence" showed reduced general functioning. This may be due to their self-esteem being low, which is related to impaired ego functioning (Bellak, Hurvich, \& Gediman, 1973), causing the individuals to lose their capacity to deal with daily activities.

\section{The Absence of a Significant Impact of "Assertion of Autonomy" on Interpersonal NLEs, and Its Impact on Dysphoric Mood and Close Functioning}

As noted, "assertion of autonomy" can be regarded as a defense against "emotional reliance," thus functioning as a strategy to avoid interpersonal NLEs that are followed by dysphoric mood. However, as noted in the introduction, it is conceivable that individuals who assert their autonomy will invite the antipathy of others. However, the dispositions of these individuals do not take into account others' feelings, and therefore they might be more likely to disregard interpersonal NLEs.

Furthermore, "assertion of autonomy" directly alleviated anxiety. While this may be interpreted as a positive occurrence, it can also be interpreted in a different way: it is possible that an individual who does not report anxiety is simply denying its existence. An individual with a high level of "assertion of autonomy" may lack the ability to recognize their own weakness and may be prone to using reaction formation (Freud, 1926) as a defense mechanism. Although "assertion of autonomy" did 
not provoke interpersonal NLEs or dysphoric mood, it lowered close functioning. Individuals who cannot respect or seek help from others because of a fear of rejection cannot establish trustworthy or intimate relationships.

\section{The Impact of Gender on the Pathways}

The only difference between males and females was the impact of interpersonal NLEs on anxiety. This means that even though males experienced interpersonal NLEs, they did not necessarily become as anxious as females. This can be interpreted that males tend to avoid recognizing anxious feelings caused by interpersonal conflicts.

\section{Application to Clinical Settings}

When we see patients who suffer from depressive mood, anxiety, or other mental symptoms, it is crucial to evaluate their personalities. In particular, the cognitive patterns, attitudes, and behaviors of patients with an intense "emotional reliance" on others are apt to elicit interpersonal distresses, resulting in undermined mental health. Therefore, removal of symptoms alone is not sufficient. It is necessary to provide support enabling these patients to obtain insight into their own personalities, thus helping to prevent future relapse.

On the other hand, individuals with a high level of "assertion of autonomy" tend to repress or deny interpersonal distresses or dysphoric moods. In the same way, those with a "lack of social self-confidence" cannot recognize interpersonal distresses, and focus predominantly on low self-esteem in a masochistic way. Therefore, we have to consider the possibility of existing interpersonal conflicts and negative emotions in these individuals' psyches.

With regard to gender, females seem to be more likely to recognize their anxiety in regard to interpersonal NLEs. For males, anxious feelings are less likely to be indicators of interpersonal NLEs.

\section{Limitation}

In this study, we defined NLEs within interpersonal relationships; however, it is uncertain whether the reported NLEs were provoked by the respondents' cognitive and behavioral patterns. Therefore, further research based on face-to-face interviews or clinical records are required.

\section{Conclusion}

The results of this study showed that "emotional reliance" can cause dysphoric mood and poor functioning in a manner mediated by interpersonal NLEs. The two JIDI factors that did not provoke interpersonal NLEs, "lack of social self-confidence" and "assertion of autonomy", directly reduced "general functioning" and "close functioning". Intense dependency as well as a lack of the ability to depend on others appears likely to undermine an individual's mental health.

\section{Acknowledgements}

We would like to express deep gratitude to respondents of this study. This research received no specific grants from any funding agency in the public, commercial, or not-for-profit sectors. There is no conflict of interest to declare.

\section{REFERENCES}

Arbuckle, J. L., \& Wothke, W. (1995). Amos 4.0 user's guide. Small Waters Cooperation.

Beck, A. T. (1983). Cognitive therapy of depression: new perspectives. In P. J. Clayton, \& J. E. Barnett (Eds.), Treatment for depression: Old controversies and new approaches (pp. 265-290). New York: Raven Press.

Bellak, L., Hurvich, M., \& Gediman, H. (1973). Ch. 5: General considerations and rationale for the selection of the 12 ego functions. In Ego functions in schizophrenics, neurotics, and normals (pp. 74-79). New York: Wiley.

Bieling, P. J., Beck, A. T., \& Brown, G. K. (2000). The sociotropyautonomy scale: Structure and implications. Cognitive Therapy and Research, 24, 763-780. http://dx.doi.org/10.1023/A:1005599714224

Bolger, N., \& Schilling, E. A. (1991). Personality and problems of everyday life: The role of neuroticism in exposure and reactivity to daily stressors. Journal of Personality, 59, 355-386. http://dx.doi.org/10.1111/j.1467-6494.1991.tb00253.x

Daley, S. E., Hammen, C., Burge, D., Davila, J., Paley, B., Lindberg, N., \& Herzberg, D. S. (1997). Predictors of generation of episodic stress: A longitudinal study of late adolescent women. Journal of Abnormal Psychology, 106, 251-259. http://dx.doi.org/10.1037/0021-843X.106.2.251

Daley, S. E., Hammen, C., Davila, J., \& Burge, D. (1998). Axis II symptomatology, depression, and life stress during the transition from adolescence to adulthood. Journal of Counseling and Clinical Psychology, 66, 595-603.

http://dx.doi.org/10.1037/0022-006X.66.4.595

Davila, J., Hammen, C., Paley, B., \& Daley, S. E. (1995). Poor interpersonal problem solving as a mechanism of stress generation in depression among adolescent women. Journal of Abnormal Psychology, 104, 592-600. http://dx.doi.org/10.1037/0021-843X.104.4.592

Evans, C., Connell, J., Barkham, M., Margison, F., McGrath, G., MellorClark, J., \& Audin, K. (2002). Towards a standardized brief outcome measure: Psychometric properties and utility of the CORE-OM (clinical outcomes in routine evaluation-outcome measure). British Journal of Psychiatry, 180, 51-60. http://dx.doi.org/10.1192/bjp.180.1.51

Freud, S. (1926). Inhibitions, symptoms and anxiety. In J. Strachey, \& A. Freud (Eds.), The standard edition of the complete psychological works of Sigmund Freud (pp. 77-175). London: The Hogarth Press.

Hammen, C. (1991). Generation of stress in the course of unipolar depression. Journal of Abnormal Psychology, 100, 555-561. http://dx.doi.org/10.1037/0021-843X.100.4.555

Hirschfeld, R. M. A., Klernman G. L., Gough, H. G., Barrett, J., Korchin, S. J., \& Chodoff, P. (1977). A measure of interpersonal dependency. Journal of Personality Assessment, 41, 610-619. http://dx.doi.org/10.1207/s15327752jpa4106 6

Kendler, K. S., Thornton, L. M., \& Prescott, C. A. (2001). Gender differences in the rates of exposure to stressful life events and sensitivity to their depressogenic effects. American Journal of Psychiatry, 158, 587-593. http://dx.doi.org/10.1176/appi.ajp.158.4.587

Magnus, K., Diener, E., Fujita, F., \& Pavot, W. (1993). Extraversion and neuroticism as predictor of objective life events: A longitudinal analysis. Journal of Personality and Social Psychology, 65, 10461053. http://dx.doi.org/10.1037/0022-3514.65.5.1046

McDonald-Scott (1988). Interpersonal dependency inventory Japanese short form: Its development and statistical test. The Japanese Journal of Nursing Research, 21, 451-460. (in Japanese)

McLennan, J., \& Bates, G. W. (1993). Vulnerability to psychological distress: Empirical and conceptual distinctions between measures of neuroticism and negative affect. Psychological Report, 73, 13151323. http://dx.doi.org/10.2466/pr0.1993.73.3f.1315

Mezulis, A. H., Funasaki, K. S., Charbonneau, A. N., \& Hyde, J. S. (2010). Gender differences in the cognitive vulnerability-stress model of depression in the transition to adolescence. Cognitive Therapy and Research, 34, 501-513. http://dx.doi.org/10.1007/s10608-009-9281-7

Ormel, J., \& Wohlfarth, T. (1991). How neuroticism, long-term difficulties, and life situation change influence psychological distress: A longitudinal model. Journal of Personality and Social Psychology, 


\section{UJI ET AL.}

60, 744-755. http://dx.doi.org/10.1037/0022-3514.60.5.744

Rudolph, K. D., \& Hammen, C. (2003). Age and gender as determinants of stress exposure, generation, and reactions in youngsters: A transactional perspective. Child Development, 70, 660-667.

http://dx.doi.org/10.1111/1467-8624.00048

Simons, A. D., Angell, K. L., Monroe, S. M., \& Thase, M. E. (1993).

Cognition and life stressors in depression: Cognitive factors and the definition, rating, and generation of negative life event. Journal of Abnormal Psychology, 102, 584-591.
http://dx.doi.org/10.1037/0021-843X.102.4.584

Uji, M., Sakamoto, A., Adachi, K., \& Kitamura, T. (2012). Psychometric properties of the Japanese version of the clinical outcomes in routine evaluation-outcome measure. Comprehensive Psychiatry, 53, 600-608. http://dx.doi.org/10.1016/j.comppsych.2011.09.006

Winnicott, D. (1958). The capacity to be alone. In M. M. Khan (Ed.), The maturational process and the facilitating environment (pp. 2936). London: Horgarth Press. 